BULLETIN OF THE

AMERICAN MATHEMATICAL SOCIETY

Volume 81, Number 3, May 1975

\title{
WHEN IS A MANIFOLD A LEAF OF SOME FOLIATION?
}

\author{
BY JONATHAN D. SONDOW 1 \\ Communicated by Glen E. Bredon, February 4, 1975
}

Given a connected smooth open manifold $L$, does there exist a compact manifold $M$ and a $C^{r}$ codimension $q$ foliation of $M$ with a leaf diffeomorphic to $L$ ? Here $1 \leqslant r \leqslant \infty$. Most of our results are for $q=1$, but note that if the answer is yes for $q$ then it is yes for any $q^{\prime}>q$. Theorem 1 gives four conditions on $L$ any one of which is sufficient, and the Corollary provides interesting examples where $L$ is a surface. We have found no necessary condition in general, but Theorem 2 gives a strong necessary condition on the ends of $L$ in order that $L$ be a codimension one leaf each of whose ends has only one asymptote. Details and proofs will appear elsewhere.

THEOREM 1. L is diffeomorphic to a leaf of a $C^{r}$ codimension $q$ foliation of some compact manifold if any one of the following conditions is satisfied ( $q=1$ except possibly in condition 1.4).

1.1. $L$ is diffeomorphic to the interior of a compact manifold-withboundary ( $r=\infty$ and $L$ will be a proper leaf).

1.2. $L=L_{1} \# L_{2}$ where $L_{1}$ and $L_{2}$ are proper leaves of $C^{r}$ codimension one foliations of compact orientable manifolds.

1.3. $L=L_{1}-X$ where $L_{1}$ is a leaf of a $C^{r}$ codimension one foliation of a compact manifold with a closed transversal which intersects $L_{1}$ in $X$.

1.4. $L$ is a regular covering space of a compact manifold with covering group which has a $C^{r}$ action on a connected compact q-manifold with a free orbit. (If the orbit is discrete, the leaf $L$ will be proper.)

Recall (see e.g. [2]) that an end $\epsilon$ of a connected manifold is determined by a sequence $U_{1} \supset U_{2} \supset \ldots$ of unbounded components of the complements of compact sets such that $\bigcap_{i=1}^{\infty} \bar{U}_{i}=\varnothing$. Another such sequence $V_{1} \supset V_{2} \supset \ldots$ determines the same end if every $U_{i}$ contains some $V_{j}$. Each $U_{i}$ is called a neighborhood of $\epsilon$. Define $\epsilon$ to be boundable if it has a closed neighborhood of the form $B \times[0, \infty)$ where $B$ is a connected compact manifold.

AMS (MOS) subject classifications (1970). Primary 57D 15.

1 This work partially supported by NSF grant GP29265.

Copyright $\odot 1975$, American Mathematical Society 
COROLLARY. Every orientable 2-manifold with a finite number of ends is a proper leaf of a $C^{r}$ foliation of a compact 3-manifold, where $r=1$ or $r=\infty$ depending on whether the number of nonboundable ends is odd or even, respectively.

If $\epsilon$ is an end of a leaf $L$ of a foliation of a manifold $M$, define the asymptote set $A_{\epsilon}$ of $\epsilon$ to be $\bigcap_{i=1}^{\infty} \mathrm{Cl}\left(U_{i}\right)$, where $\epsilon$ is determined by neighborhoods $U_{1} \supset U_{2} \supset \ldots$ in $L$ and $\operatorname{Cl}\left(U_{i}\right)$ denotes the closure of $U_{i}$ in $M$. Then $A_{\epsilon}$ is a well-defined closed union of leaves and is connected if $M$ is compact. Define a leaf $L$ to be nice if $A_{\epsilon}$ is a single leaf for every end $\epsilon$ of $L$. Note that a nice leaf is proper and that $A_{\epsilon}$ is compact if $M$ is compact. Finally, say that an end $\epsilon$ of a manifold $L$ is an infinite repetition if some closed neighborhood in $L$ of $\epsilon$ is of the form $W \cup_{f} W \cup_{f} \ldots$ where $W$ is a connected compact manifold-with-boundary, $\mathrm{Bd} W$ has two components $\mathrm{Bd} \_W$ and $\mathrm{Bd}_{+} W$, and $f: \mathrm{Bd}_{+} W \rightarrow \mathrm{Bd}_{-} W$ is a diffeomorphism.

THEOREM 2. If $L$ is a nice leaf of a $C^{1}$ codimension one foliation of a compact manifold then $L$ has only a finite number of ends and each one is an infinite repetition.

The proof uses the following two theorems, of which the first is a generalization of Reeb's first stability theorem in [3] and the second is proved using the framed surgery method of [1] .

THEOREM 3. Let $M$ be a (not necessarily compact) manifold-with-(possibly empty) boundary with a codimension $q$ foliation transverse to $\mathrm{Bd} M$. Let $A$ be a compact leaf and let $D$ be a q-disk transverse to the foliation and cutting $A$ in exactly one point $x_{0}$. Suppose there exists a point $x$ in $D$ such that each element of the holonomy group of $A$ has a representative local diffeomorphism of $D$ whose domain contains $x$ and which leaves $x$ fixed. If $x$ is sufficiently closed to $x_{0}$ then the leaf through $x$ is diffeomorphic to $A$.

Theorem 4. If $h: \Pi_{1} A \rightarrow Z$ is a surjection, where $A$ is a connected compact manifold, then there exists a smooth map $\mathrm{g}: A \rightarrow S^{1}$ such that $h=g_{*}: \Pi_{1} A \longrightarrow \Pi_{1} S^{1}=\mathrm{Z}$ and for some regular value $v$ in $S^{1}$, the manifold $g^{-1}(v)$ is connected and does not separate $A$.

\section{REFERENCES}

1. W. Browder and J. Levine, Fibering manifolds over a circle, Comment. Math. Helv. 40 (1966), 153-160. MR 33 \#3309. 
2. H. Freudenthal, Über die Enden topologischer Räume und Gruppen, Math. Z. 33 (1931), 692-713.

3. G. Reeb, Sur certains propriétés topologiques des variétés feuilletées, Actualités Sci. Indust., no. 1183, = Publ. Inst. Math. Univ. Strasbourg 11, Hermann, Paris, 1952, pp. 91-154, 157-158. MR 14, 1113 .

DEPARTMENT OF MATHEMATICS, CITY COLLEGE, NEW YORK, NEW YORK 10031 